\title{
Prognostic significance of the expression of HER family members in primary osteosarcoma
}

\author{
SHENG-LIN WANG $^{1 *}$, GUANG-XIAN ZHONG $^{1 *}$, XIN-WEN WANG ${ }^{1}$, FENG-QIANG YU $^{1}$, \\ DAN-FENG WENG ${ }^{2}$, XIN-XING WANG ${ }^{2}$ and JIAN-HUA LIN ${ }^{1,3}$ \\ Departments of ${ }^{1}$ Orthopedics and ${ }^{2}$ Pathology; ${ }^{3}$ Central Laboratory, \\ The First Affiliated Hospital of Fujian Medical University, Fuzhou, Fujian 350005, P.R. China
}

Received November 23, 2017; Accepted April 25, 2018

DOI: $10.3892 / \mathrm{ol} .2018 .8931$

\begin{abstract}
The prognosis of patients with metastatic osteosarcoma is poor and has shown no significant improvement in nearly 20 years. The human epidermal growth factor (EGF) receptor (HER) family is frequently overexpressed in the majority of human carcinomas, and is involved in promoting the proliferation and survival of cancer cells. However, the role of EGFR and HER-2 expression in osteosarcoma survival remains controversial and no previous study has simultaneously investigated the association of the expression of all the four HER family members with the prognostic significance of osteosarcoma. Therefore, the present study investigated the expression levels of the complete members of the HER family in osteosarcoma specimens, as well as their associations with the clinicopathological parameters, progression-free survival (PFS) and overall survival (OS) time of patients with osteosarcoma. The expression of HER family members was detected in osteosarcoma tumor specimens from 60 patients using immunohistochemistry. The association of the expression of HER receptors in osteosarcoma with clinicopathological parameters was analyzed using $\chi^{2}$ test and Fishers exact test. Survival analyses were evaluated by Kaplan-Meier method and Cox proportional hazards regression model. Overall, $18(30 \%), 13(22 \%), 23(38 \%)$ and $19(32 \%)$ patients presented with high expression of EGFR, HER-2, HER-3 and HER-4, respectively, and the co-expression of 2, 3 and all 4 members of the HER family was observed. High expression of EGFR and HER-4 was associated with distant metastasis. High HER-3 expression was significantly associated with an advanced Enneking stage and distant metastasis. Multivariate analysis
\end{abstract}

Correspondence to: Professor Jian-Hua Lin, Department of Orthopedics, The First Affiliated Hospital of Fujian Medical University, 20 Chazhong Road, Fuzhou, Fujian 350005, P.R. China E-mail: jianhual@126.com

${ }^{*}$ Contributed equally

Key words: osteosarcoma, epidermal growth factor receptor, immunohistochemistry, prognosis, survival demonstrated that the expression of EGFR, HER-3, HER-4, EGFR/HER-3, EGFR/HER-4 and HER-3/HER-4 was an independent predictor of poor PFS and OS time in osteosarcoma patients with stage I-IIB disease. In patients with stage IIB osteosarcoma, the expression of HER-4 and EGFR/HER-4 demonstrated a more significant effect on PFS and OS time. In conclusion, therapies targeting EGFR, HER-3 and HER-4 may provide promising strategies for primary osteosarcoma.

\section{Introduction}

Osteosarcoma is the most common primary malignant bone tumor, particularly among children and adolescents (1). The estimated incidence rate is 5 per million per year. With the introduction of adjuvant and neo-adjuvant chemotherapy into multimodal therapies, the 5-year survival rate for patients with localized osteosarcoma was improved to 60-70\%. However, the 5-year survival rate for patients with metastatic disease or relapse is only $20 \%$, and the current treatment strategies have a limited efficacy (2). Therefore, it is essential to identify more efficient prognostic factors to develop innovative and promising therapeutics, so as to further improve the prognosis of patients with osteosarcoma.

It has long been established that the human epidermal growth factor (EGF) receptor (HER) family is frequently overexpressed in the majority of human carcinomas (3). Comprised of EGFR, HER-2, HER-3 and HER-4, the family makes homodimers or heterodimers to form EGFRs. Aberrant receptor activation is involved in the improvement of the proliferation and survival of cancer cells (4).

The overexpression of EGFR in $50-70 \%$ of lung, colon and breast carcinoma cases contributes to cell proliferation, cell cycle progression and survival (5). High levels of EGFR are also associated with bone metastasis (6) and a poor prognosis in human cancer cases (7). Aberrant expression of EGFR has been reported in osteosarcoma. However, EGFR immunohistochemistry studies on osteosarcoma have revealed discrepancies ranging from no prognostic value to a good clinical outcome (8-10). HER-2 has been known to be overexpressed in $20-25 \%$ of all ovarian and breast cancer cases, in $35-45 \%$ of all pancreatic adenocarcinomas and in up to $90 \%$ of colorectal carcinomas (11). Overexpression of HER-2 may serve a role in high-grade osteosarcoma (12). 
However, favorable, unfavorable and no independent prognostic significances for HER-2 expression in osteosarcoma have been detected (13-15). The prognostic value of HER-2 remains controversial, and the efficacy of HER-2-targeting therapy in the osteosarcoma patient population has not been established (16). Increased expression of HER-3 protein has been reported in $50-70 \%$ of human breast cancer cases, and it appears to be associated with tumor size, metastasis and recurrence (17). Whereas the majority of studies observed a negative expression pattern for HER-3 across osteosarcoma cell lines and tumor specimens $(18,19)$, one previous study showed that HER-4 was involved in the tumorigenicity of osteosarcoma cells as a protective factor against various extracellular apoptotic stimuli (20). The associations of HER-3 and HER-4 expression with the survival rate of osteosarcoma patients thus require further investigation.

To the best of our knowledge, thus far, there have been no comprehensive studies on the expression of all four members of the HER family and their associations with the prognosis of patients with osteosarcoma. Therefore, the aim of the present study was to investigate the expression levels of the complete members of the HER family, as well as their co-expression in osteosarcoma specimens from 60 patients. In addition, any associations of the expression of the HER family members with clinicopathological parameters, progression-free survival (PFS) and overall survival (OS) time in patients with osteosarcoma were evaluated.

\section{Patients and methods}

Patients. A total of 72 patients with primary osteosarcoma who underwent surgical resection at the Department of Orthopedics, The First Affiliated Hospital of Fujian Medical University (Fuzhou, Fujian, China) between January 1, 2008, and December 31, 2013, were selected retrospectively. Of these, 12 cases with distant metastases at diagnosis or those with defective clinical data were excluded. The present study was approved by the Institutional Review Board of The First Affiliated Hospital of Fujian Medical University, and the protocols conformed to the ethical guidelines of the Declaration of Helsinki. All participants involved in this study provided written informed consent. The patients' medical records, including sex, age at diagnosis, primary tumor size and location, histological subtype, Enneking stage (21) and distant metastasis status were reviewed. All patients received the standardized preoperative neoadjuvant chemotherapy and postoperative chemotherapy for six courses of treatment. Chemotherapy treatment was performed with doxorubicin (60-80 $\mathrm{mg} / \mathrm{m}^{2}$, intravenous drip for 3 days), cisplatin $\left(100 \mathrm{mg} / \mathrm{m}^{2}\right.$, intravenous drip over one day) and ifosfamide $\left(8-12 \mathrm{mg} / \mathrm{m}^{2}\right.$, intravenous drip for 5 days) twice prior to the operation and 4 times following the operation. Ifosfamide was replaced with methotrexate $\left(8-12 \mathrm{mg} / \mathrm{m}^{2}\right.$, intravenous drip over one day) if the tumor necrosis rate was $<90 \%$. The 60 formalin-fixed and paraffin-embedded surgical tumor samples were obtained from the archives of the Department of Pathology (The First Affiliated Hospital of Fujian Medical University) for immunohistochemical staining.

The study cohort consisted of 39 men and 21 women with a mean age of 24 years (range, 4-55 years). A total of
25 patients presented with a primary tumor size of $<5 \mathrm{~cm}$ and 35 patients with a tumor size of $\geq 5 \mathrm{~cm}$. Stage I-IIA disease was diagnosed in 16 tumors, and stage IIB in 44 tumors, according to the Enneking staging system. The location of the tumor was the distal femur in 22 patients; shaft of femur in 9 patients; proximal tibia and humerus in 6 patients each; pelvis and distal radius in 4 patients; and proximal femur, scapula and maxilla in 3 patients. Histological subtypes were osteoblastic in 41 tumors, chondroblastic in 8, fibroblastic in 6 and special types including telangiectatic in 3 and small cell type in 2 . Subsequent to surgical resection, all patients were monitored by X-ray, lung CT scans and/or bone scanning every 3 months during the first 3 years and every 6 months thereafter, in order to evaluate the development of local recurrence and distant metastases. PFS time was defined as the interval between the date of diagnosis and first tumor progression or the last follow-up. OS time was defined as the interval between diagnosis and mortality or the last follow-up. The mean follow-up time was 44.9 months (range, 13-86 months). Among the 60 patients, 21 succumbed to osteosarcoma, 27 patients showed no evidence of disease and 12 remained alive with disease at the last follow-up.

Immunohistochemistry. Archival osteosarcoma specimens resected following neoadjuvant chemotherapy were examined for the expression of all four HER family members by immunohistochemical analysis, and 60 corresponding osteochondroma tissues were used as controls. All specimens were fixed in $10 \%$ formalin for $24-48 \mathrm{~h}$ at room temperature, embedded in paraffin, serially sectioned (4- $\mu \mathrm{m}$ thick), and stained with hematoxylin (room temperature for $10 \mathrm{~min}$ ) and eosin (room temperature for $1 \mathrm{~min}$ ) for histological observation under a light microscope (magnification, x200). The PV9000 immunohistochemical kit (Origene Technologies, Inc., Beijing, China) was used to perform the two-stage immunohistochemical method. Subsequent to incubation for $1 \mathrm{~h}$ at $60^{\circ} \mathrm{C}$, the tissue sections were deparaffinized, dehydrated and incubated with 3\% hydrogen peroxide for $10 \mathrm{~min}$ at room temperature to block endogenous peroxidase activity. During antigen retrieval process, the sections were microwaved in citrate buffer ( $\mathrm{pH} \mathrm{6.0)} \mathrm{for} 2$ min and then naturally cooled to room temperature. The sections were then immunostained with anti-EGFR (rabbit monoclonal EP38Y; cat no. ab52894; 1:50 dilution), anti-HER-2 (mousemonoclonalHRB2/451; cat no. ab187288; 1:100 dilution), anti-HER-3 (mouse monoclonalRTJ2; cat no. ab20161; 1:50 dilution) or anti-HER-4 (mouse monoclonal5G6B4; cat. no. ab204959; 1:50 dilution) (all Abcam, Cambridge, MA, USA) and incubated overnight at $4^{\circ} \mathrm{C}$. Next, the sections were incubated with Polymer Helper reagent (Origene Technologies, Inc.) for $20 \mathrm{~min}$ at $37^{\circ} \mathrm{C}$ and rinsed with phosphate-buffered saline (PBS). The sections were then incubated with poly peroxidase-anti-mouse/rabbit IgG (part of the PV9000 kit; ready-to-use dilution) for $20 \mathrm{~min}$ at room temperature. Subsequent to being washed again, the sections were stained with diaminobenzidine (both Origene Technologies, Inc.) for 3-5 min at room temperature, counterstained with hematoxylin for $2 \mathrm{~min}$ at room temperature, dehydrated, and mounted. Negative (PBS rather than primary antibodies) and known positive controls (esophagus carcinoma tissue for EGFR; breast carcinoma tissue for HER-2; 
Table I. Comparisons of HER family expression in osteosarcoma $(n=60)$ and corresponding osteochondroma $(\mathrm{n}=60)$ cases .

\begin{tabular}{|c|c|c|c|}
\hline HER family member & Osteosarcoma, n (\%) & Osteochondroma, n (\%) & P-value \\
\hline EGFR & $18(30)$ & $2(3)$ & $<0.001$ \\
\hline HER-2 & $13(22)$ & $1(0)$ & 0.002 \\
\hline HER-3 & $23(38)$ & $7(13)$ & 0.004 \\
\hline HER-4 & $19(32)$ & $2(3)$ & $<0.001$ \\
\hline EGFR/HER-2 & $6(10)$ & $0(0)$ & 0.027 \\
\hline EGFR/HER-3 & $10(17)$ & $0(0)$ & 0.001 \\
\hline EGFR/HER-4 & $5(8)$ & $0(0)$ & NS \\
\hline HER-2/HER-3 & $6(10)$ & $0(0)$ & 0.027 \\
\hline HER-2/HER-4 & $4(7)$ & $0(0)$ & NS \\
\hline HER-3/HER-4 & $7(12)$ & $0(0)$ & 0.013 \\
\hline EGFR/HER-2/HER-3 & $3(5)$ & $0(0)$ & NS \\
\hline EGFR/HER-2/HER-4 & $1(2)$ & $0(0)$ & NS \\
\hline EGFR/HER-3/HER-4 & $2(3)$ & $0(0)$ & NS \\
\hline HER-2/HER-3/HER-4 & $3(5)$ & $0(0)$ & NS \\
\hline EGFR/HER-2/HER-3/HER-4 & $1(2)$ & $0(0)$ & NS \\
\hline
\end{tabular}

NS, no significance; HER, human epidermal growth factor receptor.

oral carcinoma tissue for HER-3 and HER-4) were stained in parallel with each set of sections studied.

Observation indices and result determination. Two pathologists participated in the staining assessment of tumor specimens independently. EGFR-positive and HER-2-positive cells were those with specific brown particles in the cytoplasm or cytomembrane. Cells with brown-stained cytoplasmic or nuclear particles were determined as HER-3-positive or HER-4-positive cells. Expression was scored according to the intensity of staining of tumor cells from 0 to 3 as follows: 0 , negative; 1 , weakly positive; 2 , moderately positive; and 3 , strongly positive. The mean percentage from 10 random high-power fields of staining of positive tumor cells was also scored from 1 to 3 as follows: 1, <25\%; 2, 25-75\%; and $3,>75 \%$. For statistical analysis, the final score was calculated by the product of the density and the percentage of positive staining tumor cells, including scores $0,1,2,3,4,6$ and 9. Scores $>2$ were defined as high expression, while scores $\leq 2$ were considered as low expression (22).

Statistical analysis. The associations between HER family expression and clinicopathological parameters, including sex, age, tumor size, tumor location, histological subtype, Enneking stage and distant metastasis, were analyzed using the $\chi^{2}$ test and Fisher's exact test. The correlation between the expression of HER family members was investigated using Spearman's rank coefficient, and the difference between them was assessed by Friedman test, with the box-plot obtained using GraphPad Prism 5.0 (GraphPad Software Inc., San Diego, CA, USA). Survival analysis was performed using the Kaplan-Meier method, and differences in survival distributions were compared by the log-rank test. The Cox proportional hazards regression model was adopted to perform multivariate survival analysis for the expression of HER family members that was found to be significant in the univariate analysis (23). All statistical analyses were performed using SPSS 19.0 software (IBM, Corp., Armonk, $\mathrm{NY}$, USA). $\mathrm{P}<0.05$ was considered to indicate a statistically significant difference.

\section{Results}

Immunohistochemical expression of HER family members in osteosarcoma. Of the 60 osteosarcoma patients,18 (30\%), $13(22 \%), 23(38 \%)$ and $19(32 \%)$ cases presented with EGFR, HER-2, HER-3 and HER-4 high expression, respectively, the expression rates of which were significantly higher than those of osteochondroma (Table I). EGFR was predominantly membranous, with some cytoplasmic staining. HER-2 demonstrated a cytoplasmic staining pattern. HER-3 and HER-4 demonstrated nuclear and cytoplasmic immunostaining (Fig. 1). Fig. 1I shows the median score of HER expression (the horizontal line), the inter-quartile range (the box), and the minimum and maximum values (the whiskers) (24). Since the final scores, including 0, 1, 2, 3, 4, 6 and 9, were of skewed distribution, large error bars are present in this figure. There was no significant difference among the expression levels of the HER family members (P $>0.05$, Friedman test). The present study investigated the co-expression of 2, 3 and all 4 members of the HER family in osteosarcoma cases, which were not found in osteochondroma cases. Among which, the expression rates of EGFR/HER-2, EGFR/HER-3, HER-2/HER-3 and HER-3/HER-4 between the two groups were statistically different. EGFR, HER-2, HER-3 and HER-4 were all expressed together in only 1 case of osteosarcoma (Table I). There was no correlation among the expression levels of the HER family members, as determined using the Spearman's rank correlation in this study (Table II). 
Table II. Correlations among HER family members for patients with osteosarcoma.

\begin{tabular}{|c|c|c|c|c|c|c|}
\hline \multirow{2}{*}{$\begin{array}{l}\text { HER } \\
\text { family member }\end{array}$} & \multicolumn{2}{|l|}{ EGFR } & \multicolumn{2}{|l|}{ HER-3 } & \multicolumn{2}{|l|}{ HER-4 } \\
\hline & Correlation coefficient & P-value & Correlation coefficient & P-value & Correlation coefficient & P-value \\
\hline HER-2 & 0.185 & 0.156 & 0.085 & 0.520 & -0.010 & 0.939 \\
\hline HER-3 & 0.232 & 0.075 & - & - & -0.021 & 0.874 \\
\hline HER-4 & -0.055 & 0.678 & -0.021 & 0.874 & - & - \\
\hline
\end{tabular}

HER, human epidermal growth factor receptor.
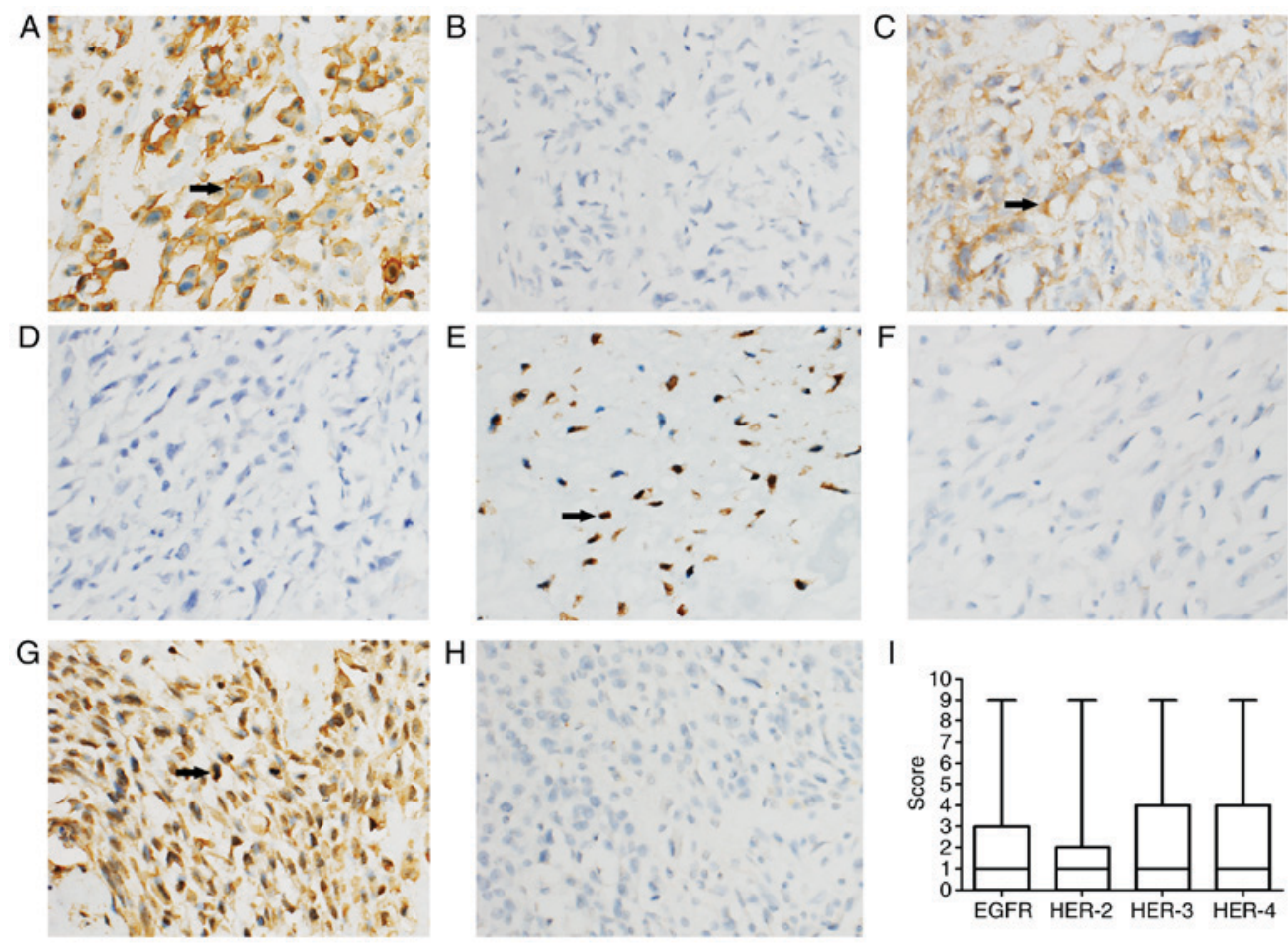

Figure 1. Immunohistochemical expression of HER family members in osteosarcoma specimens. (A) High EGFR expression: Predominantly membranous with some cytoplasmic brown staining (arrow). (B) Low EGFR expression. (C) High HER-2 expression: Predominantly cytoplasmic brown staining (arrow). (D) Low HER-2 expression. (E) High HER-3 expression: Predominantly nuclear and cytoplasmic brown staining (arrow). (F) Low HER-3 expression. (G) High HER-4 expression: Predominantly nuclear and cytoplasmic brown staining (arrow). (H) Low HER-4 expression. (I) Final HER expression score demonstrating no significant difference among the expression levels of the HER family members. The box-plot was obtained using GraphPad Prism 5.0 (GraphPad Software Inc., San Diego, CA, USA) (original magnification of Fig. A-H, x200). HER, human epidermal growth factor receptor.

Association between HER family member expression and clinicopathological characteristics. The results of the associations between HER family member expression and clinicopathological characteristics are summarized in Table III. The high expression of EGFR $(\mathrm{P}=0.027)$ and HER-4 $(\mathrm{P}=0.013)$ were associated with distant metastasis. HER-3 high expression was significantly associated with an advanced Enneking stage $(\mathrm{P}=0.029)$ and distant metastasis $(\mathrm{P}=0.013)$. Other statistically significant associations between HER family member expression and the remaining clinicopathological parameters were not found in this study.

Impact of HER family member expression on osteosarcoma patient survival. To evaluate whether the prognostic ability of HER family members was affected by clinicopathological features, univariate and multivariate analyses were performed. Results among stage I-IIB patients $(\mathrm{n}=60)$ showed that high expression of EGFR (PFS, $\mathrm{P}=0.033$; OS, $\mathrm{P}=0.041$; Fig. 2A and B), HER-3 (PFS, P=0.001; OS, P=0.006; Fig. 2C and D), HER-4 (PFS, $\mathrm{P}=0.010$; OS, $\mathrm{P}=0.015$; Fig. 2E and F), EGFR/HER-3 (PFS, $\mathrm{P}=0.010$; OS, $\mathrm{P}=0.003$ ), EGFR/HER-4 (PFS, $\mathrm{P}=0.033$; OS, $\mathrm{P}=0.033$ ), HER-2/HER-4 (PFS, $\mathrm{P}=0.001$; OS, $\mathrm{P}=0.003$ ) and HER-3/HER-4 (PFS, $\mathrm{P}<0.001$; OS, $\mathrm{P}<0.001$ ), in addition to tumor size, surgical stage and distant metastasis, were associated with short PFS and OS time upon univariate analysis (Table IV). Upon multivariate analysis with adjustment for tumor size, surgical stage and distant metastasis, the levels of EGFR, HER-3, HER-4, EGFR/HER-3, EGFR/HER-4 and HER-3/HER-4 were found to be independent predictors of poor PFS and OS time of osteosarcoma patients (Table IV). In patients with stage IIB disease only $(n=44)$, which was the predominant surgical stage of primary 
Table III. Associations between HER family member expression and clinicopathological characteristics.

\begin{tabular}{|c|c|c|c|c|c|c|c|c|c|c|c|c|c|}
\hline \multirow[b]{2}{*}{$\begin{array}{l}\text { Clinicopathological } \\
\text { data }\end{array}$} & \multirow[b]{2}{*}{ Cases, $\mathrm{n}$} & \multicolumn{3}{|c|}{ EGFR } & \multicolumn{3}{|c|}{ HER-2 } & \multicolumn{3}{|c|}{ HER-3 } & \multicolumn{3}{|c|}{ HER-4 } \\
\hline & & $\begin{array}{c}\mathrm{HE} \\
(\mathrm{n}=18)\end{array}$ & $\begin{array}{c}\mathrm{LE} \\
(\mathrm{n}=42)\end{array}$ & P-value & $\begin{array}{c}\mathrm{HE} \\
(\mathrm{n}=13)\end{array}$ & $\begin{array}{c}\mathrm{LE} \\
(\mathrm{n}=47)\end{array}$ & P-value & $\begin{array}{c}\mathrm{HE} \\
(\mathrm{n}=23)\end{array}$ & $\begin{array}{c}\mathrm{LE} \\
(\mathrm{n}=37)\end{array}$ & P-value & $\begin{array}{c}\mathrm{HE} \\
(\mathrm{n}=19)\end{array}$ & $\begin{array}{c}\mathrm{LE} \\
(\mathrm{n}=41)\end{array}$ & P-value \\
\hline \multicolumn{14}{|l|}{ Sex } \\
\hline Male & 39 & 9 & 30 & NS & 9 & 30 & NS & 13 & 26 & NS & 11 & 28 & NS \\
\hline Female & 21 & 9 & 12 & & 4 & 17 & & 10 & 11 & & 8 & 13 & \\
\hline \multicolumn{14}{|l|}{ Age, years } \\
\hline$<18$ & 26 & 9 & 17 & NS & 5 & 21 & NS & 11 & 15 & NS & 7 & 19 & NS \\
\hline$\geq 18$ & 34 & 9 & 25 & & 8 & 26 & & 12 & 22 & & 12 & 22 & \\
\hline \multicolumn{14}{|l|}{ Tumor size, $\mathrm{cm}$} \\
\hline$<5$ & 25 & 6 & 19 & NS & 4 & 21 & NS & 9 & 16 & NS & 5 & 20 & NS \\
\hline$\geq 5$ & 35 & 12 & 23 & & 9 & 26 & & 14 & 21 & & 14 & 21 & \\
\hline \multicolumn{14}{|l|}{ Tumor location } \\
\hline Tibia/Femur & 40 & 9 & 31 & NS & 9 & 31 & NS & 16 & 24 & NS & 13 & 27 & NS \\
\hline Other location & 20 & 9 & 11 & & 4 & 16 & & 7 & 13 & & 6 & 14 & \\
\hline \multicolumn{14}{|l|}{ Histological subtype } \\
\hline Conventional & 55 & 16 & 39 & NS & 12 & 43 & NS & 21 & 34 & NS & 18 & 37 & NS \\
\hline Special & 5 & 2 & 3 & & 1 & 4 & & 2 & 3 & & 1 & 4 & \\
\hline \multicolumn{14}{|l|}{ Surgical stage } \\
\hline I-IIA & 16 & 4 & 12 & NS & 3 & 13 & NS & 3 & 13 & 0.029 & 4 & 12 & NS \\
\hline IIB & 44 & 14 & 30 & & 10 & 34 & & 20 & 24 & & 15 & 29 & \\
\hline \multicolumn{14}{|l|}{ Distant metastasis } \\
\hline Yes & 27 & 12 & 15 & 0.027 & 9 & 18 & NS & 15 & 12 & 0.013 & 13 & 14 & 0.013 \\
\hline No & 33 & 6 & 27 & & 4 & 29 & & 8 & 25 & & 6 & 27 & \\
\hline
\end{tabular}

HER, human epidermal growth factor receptor; HE, high expression; LE, low expression; NS, no significance.

osteosarcoma, univariate analysis demonstrated that EGFR $(\mathrm{P}<0.001)$, HER-3 ( $\mathrm{P}=0.002)$, HER-4 ( $\mathrm{P}=0.002$; Fig. 3A), EGFR/HER-3 ( $\mathrm{P}<0.001)$, EGFR/HER-4 ( $<<0.001$; Fig. 3B), HER-2/HER-4 ( $\mathrm{P}=0.007)$ and HER-3/HER-4 $(\mathrm{P}<0.001)$, as well as tumor size and distant metastasis were associated with worse PFS time. Finally, upon multivariate analysis, EGFR, HER-4, EGFR/HER-3 and EGFR/HER-4 showed greater effects on PFS time in osteosarcoma patients with stage IIB than stage I-IIB (Table IV). With regard to OS time, EGFR ( $\mathrm{P}=0.001)$, HER-4 ( $\mathrm{P}=0.011$; Fig. 3C), EGFR/HER-3 $(\mathrm{P}=0.004)$, EGFR/HER-4 ( $\mathrm{P}<0.001$; Fig. 3D), HER-2/HER-4 $(\mathrm{P}=0.022)$ and HER-3/HER-4 $(\mathrm{P}=0.003)$ were also significant predictors in univariate analysis. Multivariate analysis suggested that HER-4, EGFR/HER-4, and HER-3/HER-4 had greater effects on OS time in osteosarcoma patients with IIB than stage I-IIB (Table IV). Therefore, expression of HER-4 and EGFR/HER-4 demonstrated greater impacts on PFS and OS time in patients with stage IIB osteosarcoma. In this study, HER-2, EGFR/HER-2 and HER-2/HER-3 demonstrated no prognostic significance among stage I-IIB and stage IIB patients upon univariate analysis (data not shown), thus were not included in multivariate analysis demonstrated in Table IV. The potential disadvantage of this approach was that variables with $\mathrm{P}>0.05$ in univariate analysis may finally be significant in the further multivariate analysis, as demonstrated by a previous study (25). However, the three aforementioned receptors were verified to exhibit no statistical significance upon a different multivariate analysis including all variables (data not shown). Consequently, the survival analyses did not cause bias to the results.

\section{Discussion}

Previous studies on EGFR and HER-2 expression in osteosarcoma have created controversy over their roles in osteosarcoma survival $(9,10,14,15)$. Furthermore, no previous study has simultaneously investigated the associations between the expression of all four members of the HER family and their prognostic significance in osteosarcoma. In the present study, the high expression levels of EGFR, HER-2, HER-3 and HER-4, and the co-expression of 2, 3 and all 4 members of the HER family were identified. Furthermore, EGFR, HER-3 and HER-4 expression, as well as the expression of EGFR/HER-3, EGFR/HER-4 and HER-3/HER-4 were found to be independent prognostic factors of poor PFS and OS time in stage I-IIB osteosarcoma. The expression of HER-4 and EGFR/HER-4 had more significant impacts on PFS and OS time in osteosarcoma patients with stage IIB disease than in those with stage I-IIB.

The present results showed high EGFR expression with membranous and some cytoplasmic staining, which 

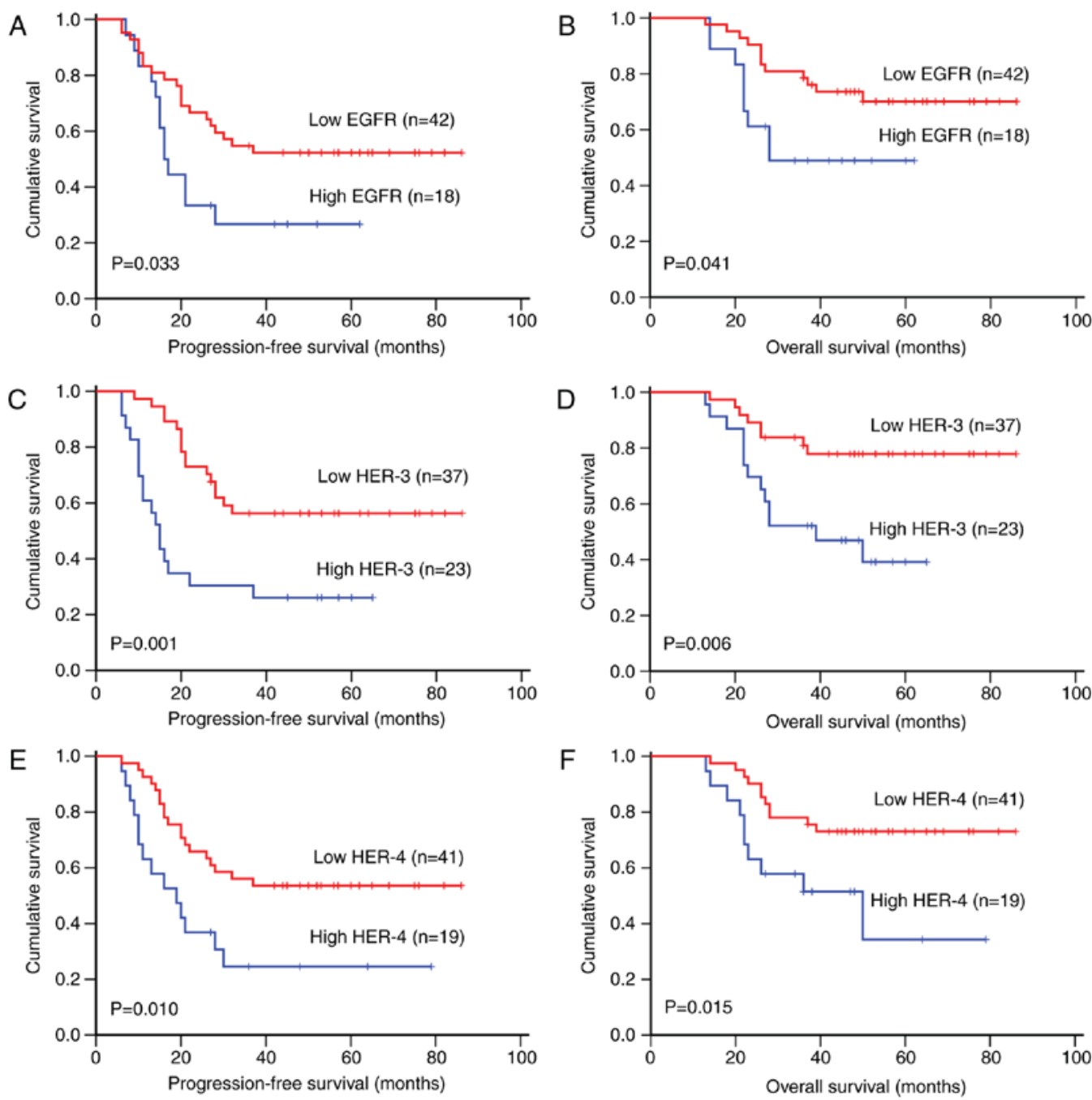

Figure 2. Kaplan-Meier analyses of PFS and OS time in stage I-IIB osteosarcoma patients. Significant differences in (A) PFS (P=0.033) and (B) OS (P=0.041) time were found between the high and low EGFR expression groups. Significant differences in (C) PFS $(P=0.001)$ and $(D)$ OS $(P=0.006)$ time were found between the high and low HER-3 expression groups. Significant differences in (E) PFS ( $\mathrm{P}=0.010)$ and $(\mathrm{F}) \mathrm{OS}(\mathrm{P}=0.015)$ time were found between the high and low HER-4 expression groups. HER, human epidermal growth factor receptor; PFS, progression-free survival; OS, overall survival.

was significantly associated with poor survival, indicating the involvement of EGFR in the development of osteosarcoma. Probably due to the small sample size and selection bias, studies by Do et al (8) and Lee et al (9) did not find a similar association. Kersting et al (10) observed an association between EGFR expression and a favorable clinical outcome. However, the efficacy of EGFR-targeting agents (cetuximab) in the management of osteosarcoma does not support this conclusion (26). Interplay of heterodimers of the HER may not explain the unexpected finding either, since EGFR/HER-3 and EGFR /HER-4 were found to be associated with the poor survival of patients with osteosarcoma in the present study. Tumors initially sensitive to anti-EGFR agent, such as cetuximab, often develop resistance. Compensatory HER-3/PI3K/AKT signaling has been confirmed as vital in the development of acquired resistance to EGFR inhibitors (27). Dimerization of HER-4 with EGFR is a crucial step to stimulate the HER-4 receptor. Interactions between EGFR and HER-4 regulate stretch-induced differentiation of fetal lung cells via the ERK pathway (28). In addition, a previous study showed that co-expression of EGFR and HER-4 contributed to neoplastic transformation, and was demonstrated to predict the invasion and poor clinical outcomes of oral squamous cell carcinoma (29). The present results supported the potential of EGFR within dual targeting therapy, such as combined treatment with MEHD7945A (27), for refractory osteosarcoma in the future.

The expression rate of HER-2 ranges from its detection in 4 to $71.9 \%$ of patients $(12,15,30)$ to the absence of expression (31), as determined by previous studies. These conflicting results may be due to differences in technical method, specimen treatment, antibodies used or results interpretation. The present results revealed a HER-2 cytoplasmic staining pattern in $13(22 \%)$ osteosarcoma samples, with no prognostic significance, which is supported by previous studies $(13,32)$. Scotlandi et al (33) observed no therapeutic effectiveness for trastuzumab-driven therapy in osteosarcoma. Since trastuzumab targets the extracellular domain of HER-2, incomplete membranous immunoreactivity for HER-2 may induce resistance to trastuzumab-driven therapy (34). Lapatinib potently and reversibly binds to the intracellular domains of HER-2, and has been proven to alter the malignant phenotype 


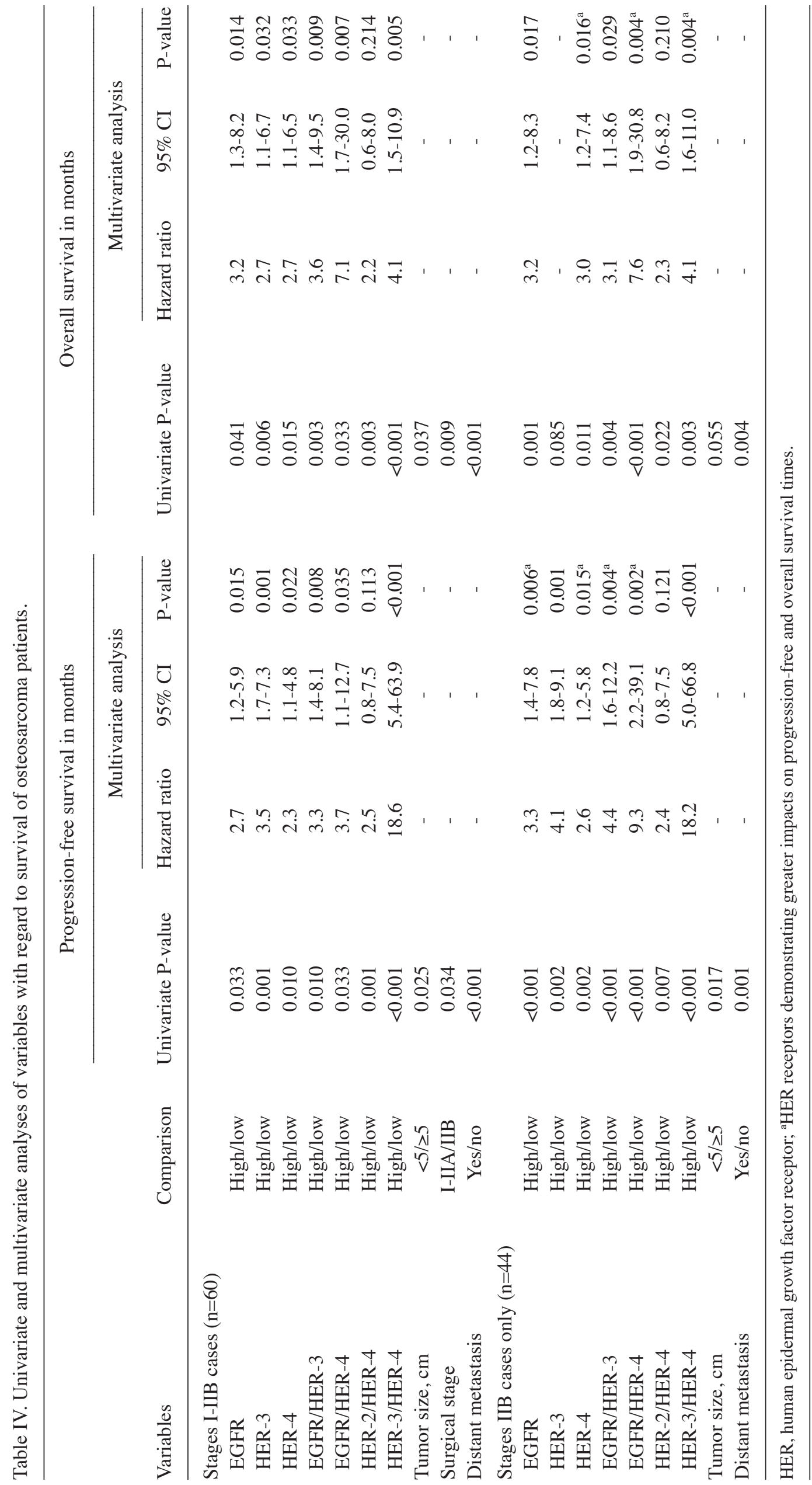



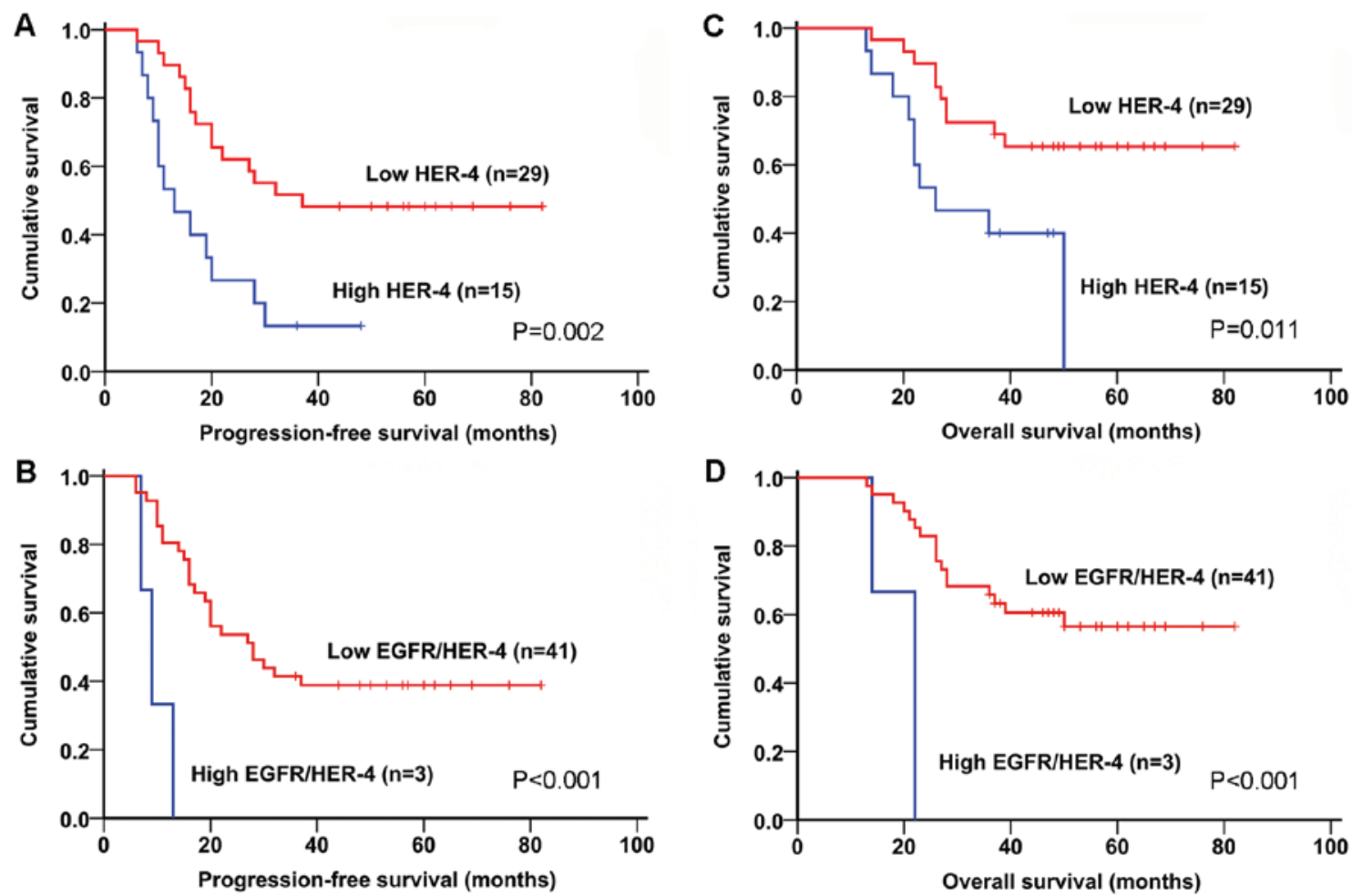

Figure 3. Kaplan-Meier analyses of PFS and OS time in stage IIB osteosarcoma patients. Significant differences of PFS time were found between the high and low (A) HER-4 ( $\mathrm{P}=0.002)$ expression groups and (B) EGFR/HER-4 ( $\mathrm{P}<0.001)$ expression groups. Significant differences of OS time were found between the high and low (C) HER-4 ( $\mathrm{P}=0.011)$ expression groups and (D) EGFR/HER-4 ( $\mathrm{P}<0.001)$ expression groups. HER, human epidermal growth factor receptor; PFS, progression-free survival; OS, overall survival.

of osteosarcoma cells (35). Thus, lapatinib is supported as a promising chemotherapeutic agent for the treatment of osteosarcoma. Nevertheless, the correlation between the expression of HER-2 and cancer regulation molecules, including tumor protein $\mathrm{p} 53$ or retinoblastoma protein, and associated signaling pathways should be confirmed in future studies.

In the present study, HER-3 demonstrated high expression in 23 cases (38\%), with a nuclear and cytoplasmic staining pattern, which is different from the majority of previous studies observing negative expression for HER-3 in osteosarcoma cell lines and tumor specimens $(18,19)$. The unfavorable prognostic role of HER-3 expression in osteosarcoma investigated in the present study may be explained by the two following mechanisms. Firstly, downregulation of HER-3 contributes to early osteoblast differentiation of mesenchymal stem cells through increased Wnt $/ \beta$-catenin signaling (36). However, the absence of nuclear $\beta$-catenin staining and Wnt-luciferase activity have been found in the biopsies and cell lines of osteosarcoma. Therefore, HER-3 overexpression-induced inactivation of the Wnt/ $\beta$-catenin pathway activity, which is required for osteoblast differentiation, may contribute to osteosarcoma development (37). Secondly, overexpression of microRNA (miR)-3928, which targets the HER-3 gene, induces cell apoptosis and inhibits tumor growth. HER-3 overexpression induced by the downregulated expression of miR-3928 in osteosarcoma may be another factor promoting cell proliferation and tumor growth (38). HER-3/HER-4 expression was also found to be significantly associated with worse PFS and OS time in the present study. HER-3 and HER-4 were significantly co-expressed in a large fraction of tumors (39) and the co-expression pattern may represent a crucial intracellular molecular switch in breast carcinogenesis (40). Agents trapping HER-3 in the inactive conformation may provide an effective therapeutic strategy for osteosarcoma patients.

Nuclear localization of HER-4 antigen in osteosarcoma tumor specimens in the present study was generally concordant with the protein expression measured by Hughes et al (19). The cleavage of HER-4 by ligand binding leads to a Mr 80,000 fragment of HER-4 translocation to the nucleus (41). The nuclear pattern of HER-4 expression observed by the present study suggested an activated state. Upregulated HER-4 expression in non-adherent tumor spheroids of osteosarcoma contributes to the tolerance to anoikis, serum starvation and chemotherapy resistance via regulating the survival pathway of osteosarcoma under various cellular stress conditions (20). HER-4 signaling is the key backup mechanism contributing to acquired resistance to HER-targeted therapies, and knockdown of HER-4, but not HER1-3, led to apoptosis in resistant breast cancer cells (42). The noticeable adverse effect of HER-4 expression on the survival of osteosarcoma patients with stage I-IIB and stage IIB disease investigated in the present study confirms the significant contribution of HER-4 to osteosarcoma pathogenesis and outcome. Thus, pan-HER, particularly HER-4-targeted inhibitors, may provide an insight into the treatment of resistant osteosarcoma.

Certain limitations should be considered in the present study. First, due to the retrospective nature of this study, selection bias may have existed when collecting patient information; a possibly randomized and prospective study would 
further clarify the prognostic role of HER receptors in osteosarcoma. Second, the application of immunohistochemistry and semi-quantitative measures for the assessment of HER expression is somewhat subjective and imposes limitations. The present findings should promote further investigation into the quantitative analysis of the receptors and associated molecular mechanisms using fresh osteosarcoma specimens or cell lines. Third, the study cohort included a relatively small number of patients, and the follow-up to evaluate the patient survival was relatively short; further large-scale research and a longer follow-up time would offer more convincing evidence in the future.

In conclusion, the present study demonstrated that the expression of EGFR, HER-3 and HER-4 together with the heterodimerization of EGFR/HER-3, EGFR/HER-4 and HER-3/HER-4 may contribute significantly to the unfavorable clinical outcome of osteosarcoma patients. Therapies targeting EGFR, HER-3 and HER-4 may provide promising strategies for treating primary osteosarcoma, if these results are confirmed by larger multicenter clinical studies.

\section{Acknowledgements}

The authors would like to thank Dr Chen Yupeng and Dr Zhang Zhenzhen from Department of Pathology, The First Affiliated Hospital of Fujian Medical University for providing instruction with regard to the immunohistochemistry protocols.

\section{Funding}

This study was supported by the National Natural Science Foundation of China (grant no. 31571292), the Joint Funds for the Innovation of Science and Technology of Fujian Province (grant no. 2016Y9019) and the Outstanding Youth Fund of Fujian Province (grant no. 2017J06017).

\section{Availability of data and materials}

The datasets used and/or analyzed during the current study are available from the corresponding author on reasonable request.

\section{Authors' contributions}

SLW and GXZ conducted the experiment and drafted the manuscript. XWW and FQY contributed to statistical analysis and manuscript writing. DFW and XXW participated in collecting data. JHL conceived the present study and helped to revise the manuscript. All authors read and approved the final manuscript.

\section{Ethics approval and consent to participate}

The present study was approved by the Institutional Review Board of The First Affiliated Hospital of Fujian Medical University, and the protocols conformed to the ethical guidelines of the Declaration of Helsinki. All participants involved in the present study provided written informed consent.

\section{Consent for publication}

Patient, parent or next of kin provided written informed consent for the publication of any associated data and accompanying images.

\section{Competing interests}

The authors declare that they have no competing interests.

\section{References}

1. Mirabello L, Troisi RJ and Savage SA: Osteosarcoma incidence and survival rates from 1973 to 2004: Data from the surveillance, epidemiology, and end results program. Cancer 115: 1531-1543, 2009.

2. Zhou W, Hao M, Du X, Chen K, Wang G and Yang J: Advances in targeted therapy for osteosarcoma. Discov Med 17: 301-307, 2014.

3. De Luca A, Carotenuto A, Rachiglio A, Gallo M, Maiello MR, Aldinucci D, Pinto A and Normanno N: The role of the EGFR signaling in tumor microenvironment. J Cell Physiol 214: 559-567, 2008 .

4. Mendelsohn J and Baselga J: Epidermal growth factor receptor targeting in cancer. Semin Oncol 33: 369-385, 2006.

5. Renoir JM, Marsaud V and Lazennec G: Estrogen receptor signaling as a target for novel breast cancer therapeutics. Biochem Pharmacol 85: 449-465, 2013.

6. Siu MK, Abou-Kheir W, Yin JJ, Chang YS, Barrett B, Suau F Casey O, Chen WY, Fang L, Hynes P, et al: Loss of EGFR signaling-regulated miR-203 promotes prostate cancer bone metastasis and tyrosine kinase inhibitors resistance. Oncotarget 5: 3770-3784, 2014

7. Xu L, Wu H, Jiang C, Wang H, Gao B, Yan S, Qi Y and Zhou S: Dacomitinib, a new pan-EGFR inhibitor, is effective in killing ovarian cancer cells. Discov Med 22: 297-309, 2016.

8. Do SI, Jung WW, Kim HS and Park YK: The expression of epidermal growth factor receptor and its downstream signaling molecules in osteosarcoma. Int J Oncol 34: 797-803, 2009.

9. Lee JA, Ko Y, Kim DH, Lim JS, Kong CB, Cho WH, Jeon DG, Lee SY and Koh JS: Epidermal growth factor receptor: Is it a feasible target for the treatment of osteosarcoma? Cancer Res Treat 44: 202-209, 2012

10. Kersting C, Gebert C, Agelopoulos K, Schmidt H, van Diest PJ, Juergens H, Winkelmann W, Kevric M, Gosheger G, Brandt B, et al: Epidermal growth factor receptor expression in high-grade osteosarcomas is associated with a good clinical outcome. Clin Cancer Res 13: 2998-3005, 2007.

11. Baxevanis CN, Sotiropoulou PA, Sotiriadou NN and Papamichail M: Immunobiology of HER-2/neu oncoprotein and its potential application in cancer immunotherapy. Cancer Immunol Immunother 53: 166-175, 2004.

12. Abdou AG, Kandil M, Asaad NY, Dawoud MM, Shahin AA and Abd Eldayem AF: The prognostic role of Ezrin and HER2/neu expression in osteosarcoma. Appl Immunohistochem Mol Morphol: AIMM 24: 355-363, 2016.

13. Yalcin B, Gedikoğlu G, Kutluk T, Varan A, Akyüz C and Büyükpamukçu M: C-erbB-2 expression and prognostic significance in osteosarcoma. Pediatric Blood Cancer 51: 222-227, 2008.

14. Zhang Q, Liu F, Wang B, Li Z, Zhou D, Yang Q, Dong J and Li J: HER-2 expression in biopsy and surgical specimen on prognosis of osteosarcoma: A systematic review and meta-analysis of 16 studies. Medicine 95: e3661, 2016.

15. Akatsuka T, Wada T, Kokai Y, Kawaguchi S, Isu K, Yamashiro K, Yamashita T, Sawada N, Yamawaki S and Ishii S: ErbB2 expression is correlated with increased survival of patients with osteosarcoma. Cancer 94: 1397-1404, 2002.

16. Geller DS and Gorlick R: HER-2 targeted treatment of osteosarcoma: The challenges of developing targeted therapy and prognostic factors for rare malignancies. Exp Opin Pharmacother 11: 51-61, 2010.

17. Ma J, Lyu H, Huang J and Liu B: Targeting of erbB3 receptor to overcome resistance in cancer treatment. Mol Cancer 13: 105, 2014. 
18. Hassan SE, Bekarev M, Kim MY, Lin J, Piperdi S, Gorlick R and Geller DS: Cell surface receptor expression patterns in osteosarcoma. Cancer 118: 740-749, 2012.

19. Hughes DP, Thomas DG, Giordano TJ, Baker LH and McDonagh KT: Cell surface expression of epidermal growth factor receptor and Her-2 with nuclear expression of Her-4 in primary osteosarcoma. Cancer Res 64: 2047-2053, 2004.

20. Hua Y, Yang Y and Hughes D: Abstract 197: Multi-cellular tumor spheroids in vitro model reveals a critical role of ERBB4 in survival of osteosarcoma. Cancer Res 71: 197-197, 2011.

21. Enneking WF, Spanier SS and Goodman MA: A system for the surgical staging of musculoskeletal sarcoma. Clin Orthop Relat Res: 106-120, 1980

22. Srirajaskanthan R, Shah T, Watkins J, Marelli L, Khan K and Caplin ME: Expression of the HER-1-4 family of receptor tyrosine kinases in neuroendocrine tumours. Oncol Rep 23: 909-915, 2010.

23. Liu Q, Hao L, Lou Z, Gao X, Gong H, Hong Y, Fu C and Zhang W: Survival time and prognostic factors of patients with initial noncurative colorectal liver metastases. Medicine (Baltimore) 96: e8831, 2017.

24. Wu H, Sharp GC, Zhao Q, Shirato H and Jiang SB: Statistical analysis and correlation discovery of tumor respiratory motion. Phys Med Biol 52: 4761-4774, 2007.

25. Nishikawa H, Nishijima N, Arimoto A, Inuzuka T, Kita R, Kimura T and Osaki Y: Prognostic factors in patients with hepatitis B virus-related hepatocellular carcinoma undergoing nucleoside analog antiviral therapy. Oncol Lett 6: 1213-1218, 2013.

26. Pahl JH, Ruslan SE, Buddingh EP, Santos SJ, Szuhai K, Serra M, Gelderblom H, Hogendoorn PC, Egeler RM, Schilham MW and Lankester AC: Anti-EGFR antibody cetuximab enhances the cytolytic activity of natural killer cells toward osteosarcoma. Clin Cancer Res 18: 432-441, 2012.

27. Huang S, Li C, Armstrong EA, Peet CR, Saker J, Amler LC, Sliwkowski MX and Harari PM: Dual targeting of EGFR and HER3 with MEHD7945A overcomes acquired resistance to EGFR inhibitors and radiation. Cancer Res 73: 824-833, 2013.

28. Huang Z, Wang Y, Nayak PS, Dammann CE and Sanchez-Esteban J: Stretch-induced fetal type II cell differentiation is mediated via ErbB1-ErbB4 interactions. J Biol Chem 287: 18091-18102, 2012.

29. Silva SD, Alaoui-Jamali MA, Hier M, Soares FA, Graner E and Kowalski LP: Cooverexpression of ERBB1 and ERBB4 receptors predicts poor clinical outcome in $\mathrm{pN}+$ oral squamous cell carcinoma with extranodal spread. Clin Exp Metastasis 31: 307-316, 2014

30. Anninga JK, van de Vijver MJ, Cleton-Jansen AM, Kristel PM, Taminiau AH, Nooij M, Egeler RM and Hogendoorn PC: Overexpression of the HER-2 oncogene does not play a role in high-grade osteosarcomas. Eur J Cancer 40: 963-970, 2004.
31. Maitra A, Wanzer D, Weinberg AG and Ashfaq R: Amplification of the HER-2/ neu oncogene is uncommon in pediatric osteosarcomas. Cancer 92: 677-683, 2001.

32. Kilpatrick SE, Geisinger KR, King TS, Sciarrotta J, Ward WG, Gold SH and Bos GD: Clinicopathologic analysis of HER-2/neu immunoexpression among various histologic subtypes and grades of osteosarcoma. Mod Pathol 14: 1277-1283, 2001.

33. Scotlandi K, Manara MC, Hattinger CM, Benini S, Perdichizzi S, Pasello M, Bacci G, Zanella L, Bertoni F, Picci P and Serra M: Prognostic and therapeutic relevance of HER2 expression in osteosarcoma and Ewing's sarcoma. Eur J Cancer 41: 1349-1361, 2005.

34. Yoshida R, Tazawa H, Hashimoto Y, Yano S, Onishi T, Sasaki T, Shirakawa Y,Kishimoto H, Uno F, Nishizaki M, et al: Mechanism of resistance to trastuzumab and molecular sensitization via ADCC activation by exogenous expression of HER2-extracellular domain in human cancer cells. Cancer Immunol Immunother 61: 1905-1916, 2012

35. Long XH, Zhang GM, Peng AF, Luo QF, Zhang L, Wen HC, Zhou RP, Gao S, Zhou Y and Liu ZL: Lapatinib alters the malignant phenotype of osteosarcoma cells via downregulation of the activity of the HER2-PI3K/AKT-FASN axis in vitro. Oncol Rep 31: 328-334, 2014

36. Jullien N, Maudinet A, Leloutre B, Ringe J, Häupl T and Marie PJ: Downregulation of ErbB3 by Wnt3a contributes to wnt-induced osteoblast differentiation in mesenchymal cells. J Cell Biochem 113: 2047-2056, 2012.

37. Cai Y, Mohseny AB, Karperien M, Hogendoorn PC, Zhou G and Cleton-Jansen AM: Inactive Wnt/beta-catenin pathway in conventional high-grade osteosarcoma. J Pathol 220: 24-33, 2010.

38. Xu H, Liu X and Zhao J: Down-regulation of miR-3928 promoted osteosarcoma growth. Cell Physiol Biochem 33: 1547-1556, 2014.

39. Bianchi S, Palli D, Falchetti M, Saieva C, Masala G, Mancini B, Lupi R, Noviello C, Omerovic J, Paglierani M, et al: ErbB-receptors expression and survival in breast carcinoma: A 15-year follow-up study. J Cell Physiol 206: 702-708, 2006.

40. Karamouzis MV, Badra FA and Papavassiliou AG: Breast cancer: The upgraded role of HER-3 and HER-4. Int J Biochem Cell Biol 39: 851-856, 2007.

41. Citri A and Yarden Y: EGF-ERBB signalling: Towards the systems level. Nat Rev Mol Cell Biol 7: 505-516, 2006.

42. Canfield K, Li J, Wilkins OM, Morrison MM, Ung M, Wells W, Williams CR, Liby KT, Vullhorst D, Buonanno A, et al: Receptor tyrosine kinase ERBB4 mediates acquired resistance to ERBB2 inhibitors in breast cancer cells. Cell Cycle 14: 648-655, 2015.

This work is licensed under a Creative Commons Attribution-NonCommercial-NoDerivatives 4.0 International (CC BY-NC-ND 4.0) License. 